\title{
Na Current in Membrane Blebs: Implications for Channel Mobility and Patch Clamp Recording
}

\author{
R. L. Milton ${ }^{2, a}$ and J. H. Caldwell ${ }^{1,2}$ \\ 'Department of Cellular and Structural Biology and ${ }^{2}$ Department of Physiology, University of Colorado Health Sciences \\ Center, Denver, Colorado 80262
}

When suction was applied to loose patch clamp pipettes while recording from enzymatically dissociated muscle fibers, large membrane blebs formed within the plpettes. We initiated a study of these suction-induced blebs because ion channels in the blebs would complicate or possibly invalidate loose patch voltage clamp measurements of membrane current density. The low lateral mobility (Stühmer and Almers, 1982) and steep gradients of $\mathrm{Na}$ channels at the endplate and tendon (Caldwell et al., 1986) imply tight binding of Na channels to cytoskeletal elements and led us to expect few, if any, Na channels in the blebs.

Bleb formation produced an increase in membrane capacitance, as expected from the increase in membrane area. Bleb formation also increased the Na current, indicating that the blebs contained $\mathrm{Na}$ channels. Assuming that the increased capacitance and $\mathrm{Na}$ current were due to lipid and $\mathrm{Na}$ channels moving from membrane outside the pipette, ejection of the bleb from the pipette was expected to bring the capacitance and $\mathrm{Na}$ current back to their original values. Capacitance did return to its original value, but Na current was lower than expected. The decrease in $\mathrm{Na}$ current is explained by $\mathrm{Na}$ channels moving from the patch membrane into the bleb.

Normalization of bleb and patch $\mathrm{Na}$ current to their respective capacitances revealed that bleb membrane had a Na channel density almost $50 \%$ that of normal surface membrane. Thus, bleb membrane is neither devoid of proteins nor truly representative of the normal surface membrane from which it arose. It is enriched in membrane lipids and is relatively protein poor.

Two conclusions can be drawn. (1) Measurements of current density made with the loose patch voltage clamp may appear highly variable and be inaccurate if bleb formation occurs and is ignored. (2) A large fraction of $\mathrm{Na}$ channels in adult mouse muscle may be mobile. A model is presented to reconcile the apparent contradiction between the existence of large gradients of $\mathrm{Na}$ channel density at the endplate and tendon and the presence of mobile channels.

Received May 3, 1989; revised Aug. 14, 1989; accepted Aug. 14, 1989.

We thank Dr. W. Almers for criticisms of the manuscript and Diane Nicholl for technical assistance. This research was supported by grants from the NSF BNS 8809182 and Muscular Dystrophy Association (J.H.C.) and NIH NRSA fellowship NS 07952 (R.L.M.)

Correspondence should be addressed to Dr. John H. Caldwell, Department of Cellular and Structural Biology, University of Colorado Health Sciences Center 4200 E. Ninth Ave--Box B-111, Denver, CO 80262.

a Present address: Indiana University School of Medicine, Muncie Center for Medical Education, Ball State University, Muncie, IN 47306.

Copyright (C) 1990 Society for Neuroscience $0270-6474 / 90 / 030885-09 \$ 02.00 / 0$
Many, if not most, transmembrane proteins exhibit some degree of nonuniformity in their distributions (see reviews by Axelrod, 1983; Almers and Stirling, 1984). Although there is no clear understanding of the mechanisms that establish and maintain these heterogeneous distributions, it is evident that they require some restriction of the free diffusion of proteins within the plasma membrane. Recent measurements have revealed that many membrane proteins have diffusion coefficients of at least an order of magnitude less than would be expected from bilayer viscosity. This reduced mobility could be explained by cytoskeletal or extracellular elements acting to limit membrane protcin diffusion. This supposition is supported by measurements of protein diffusion coefficients in membrane blebs that are free of attachments to the cytoskeleton or extracellular matrix (Tank et al., 1982). These measurements revealed that acetylcholine receptors were freely mobile in blebs formed on skeletal muscle fibers and had diffusion coefficients close to that expected due to lipid bilayer viscosity, i.e., several orders of magnitude greater than in normal sarcolemma.

We report here the measurement of $\mathrm{Na}$ channel density in suction-induced membrane blebs from dissociated skeletal muscle fibers. These blebs are not formed by a simple distension of the surface membrane but result from the bulk flow of membrane lipids into the patch membrane as a consequence of the applied suction. These blebs resemble those that can be induced by a wide variety of treatments, including enzymes (Standen et al., 1984), aldehydes (Scott, 1976), and osmotic shock (Zollinger, 1948), and in particular appear similar to those studied by Tank et al. (1982) in mouse muscle. This similarity in appearance, plus the observation that blebs can grow to a diameter larger than that of the muscle fiber from which they arise without any visible distortion of the Z-lines or surrounding fiber morphology, supports the hypothesis that these blebs are likely to be free of cytoskeletal or extracellular attachments.

$\mathrm{Na}$ currents were measured from a given membrane patch in the following sequence. First, $\mathrm{Na}$ current and membrane capacitance were measured before bleb formation. Then suction was applied until a bleb formed, and the total current and capacitance were measured from the patch and bleb. Finally, suction was removed, the bleb was blown out of the pipette, and the current and capacitance were again recorded from the patch. In addition, in a few cases, $\mathrm{Na}$ current was recorded from isolated blebs.

Our findings have implications for (1) Na channel mobility and (2) use of the loose patch clamp technique. A simple model, which may help to explain our mobility results as well as those of other researchers, is presented. 

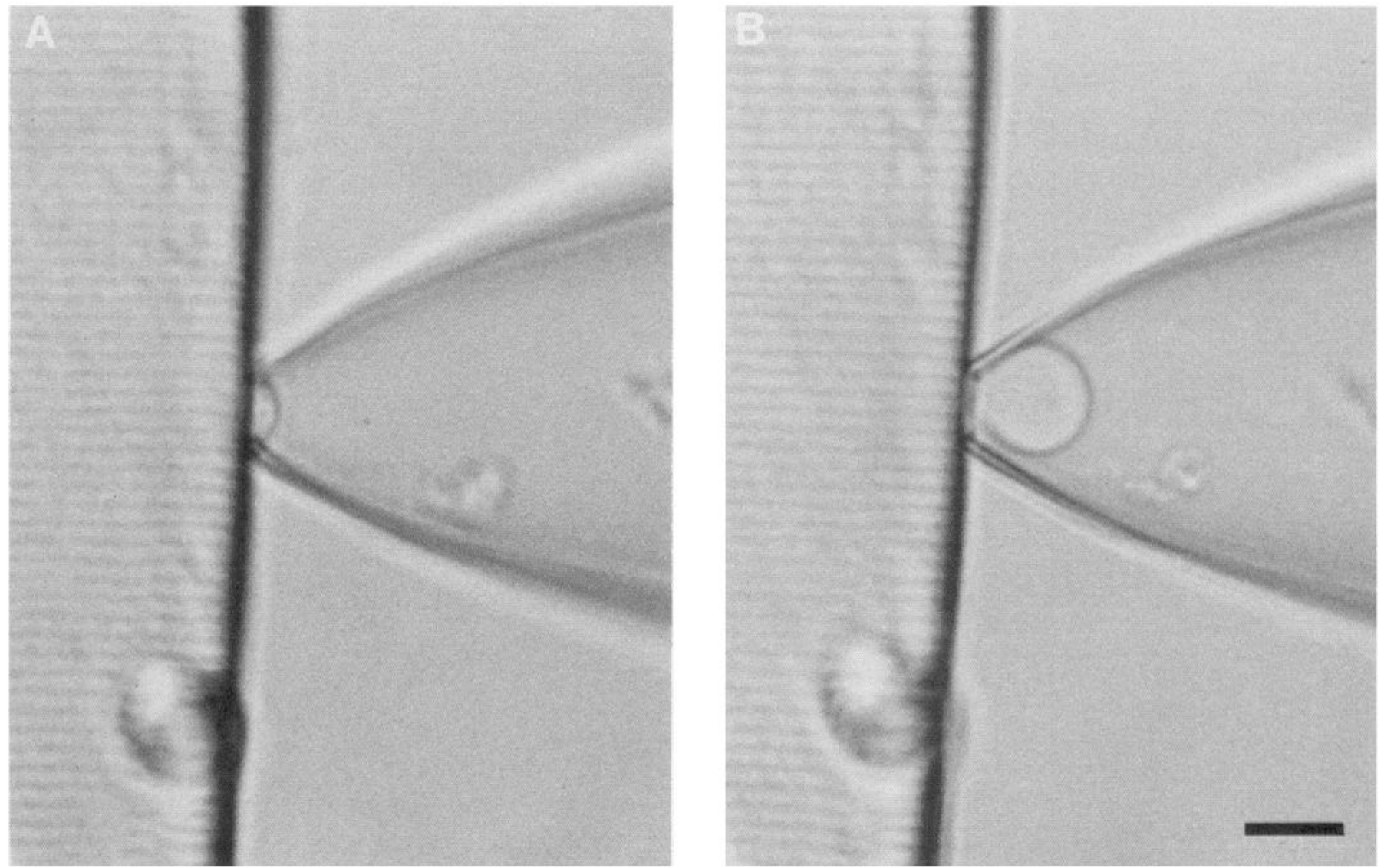

Figure 1. Loose patch pipette pressed against surface of an enzymatically dissociated mouse FDB fiber. $A$, Suction applied to the interior of the pipette caused the membrane to bulge into the pipette, but no bleb has formed. $B$, A bleb that originated at the inner rim of the pipette has formed. The patch membrane (out of focus because of a focal change between $A$ and $B$ ) was unaltered by the bleb formation. The scale bar (10 $\mu$ m) applies to both $A$ and $B$.

\section{Materials and Methods}

Preparation. The preparation used in all the experiments described was the flexor digitorum brevis (FDB) muscle of the mouse $(25-40 \mathrm{gm}$; Swiss Webster). The mice were killed with an overdose of ether, and the muscles were dissected from the animals. The muscle fibers were dissociated using a modification of the procedure described by Bekoff and Betz (1977). Briefly, the muscle was placed in a test tube containing Dulbecco's modified Eagle medium with collagenase $(2.5 \mathrm{mg} / \mathrm{ml}$, type I, Sigma Co., St. Louis, MO), and BSA (1 mg/ml, fraction V, Sigma Co., St. Louis, MO) was added. Incubation in this medium for approximately $2 \mathrm{hr}$ at $37^{\circ} \mathrm{C}$ followed by trituration with a fire-polished Pasteur pipette produced numerous dissociated single fibers.

Solutions and patch pipettes. All experiments were carried out at 20 $22^{\circ} \mathrm{C}$ in physiological Ringer's solution of the following composition (concentrations in mM): $139 \mathrm{Na}^{+}, 5 \mathrm{~K}^{+}, 2 \mathrm{Ca}^{2+}, 1 \mathrm{Mg}^{2+}, 146 \mathrm{Cl}^{-}, 11$ glucose, and 2 PIPES; $\mathrm{pH}$ adjusted to 7.4 by addition of $\mathrm{HCl}$.

Loose patch pipettes were pulled from borosilicate-glass capillary tubing (5068, Rochester Scientific Co., Rochester, NY) using a 2-step procedure as described by Strickholm (1961). All pipettes were firepolished before use to aid in seal formation. Final tip diameters varied from 8 to $18 \mu \mathrm{m}$. Pipettes were filled with normal Ringer's and had resistances ranging from $100 \mathrm{k} \Omega$ to $250 \mathrm{k} \Omega$, depending on tip diameter and taper. Before filling, pipettes were heated and bent to an angle of almost $90^{\circ}$ so that the surface of a fiber could be approached from a nearly vertical direction. A piezoelectric positioner (Burleigh Instruments, Fisher, NY) was used to give precise control over the advance of the pipette. Following contact with the fiber surface, the pipette was advanced in $2 \mu \mathrm{m}$ steps until the resistance between the interior of the pipette and the bathing solution (seal resistance) was equal to or greater than the pipette resistance. Suction $(10-80 \mathrm{~mm} \mathrm{Hg})$ could then be applied to improve seal resistance and stability. However, even small amounts of suction tended to cause the formation of blebs inside the pipette (Fig. 1; described in Results). Therefore, when such blebs were not desired, suction was not applied. The processes of seal and bleb formation were continually monitored visually by means of a television monitor (Model TC1910, RCA, Lancaster, PA) connected to a videocamera (Model TC2000, RCA, Lancaster, PA) attached to an inverting microscope equipped with Hoffman modulation contrast optics (Nikon Diaphot, Nikon, Inc., Garden City, NY).

Measurement of patch capacitance. The voltage clamp circuitry used was similar to that described by Stühmer et al. (1983), with some modifications for measurements of the capacitance of the membrane under the pipette (patch capacitance). These modifications were required to ensure accurate compensation at all times for current shunted through the seal resistance. Such accurate compensation is necessary, since even at frequencies as high as $1 \mathrm{kHz}$, approximately $99 \%$ of the current in the pipette will be resistive current through the seal and only the remaining $1 \%$ will be capacitive current across the patch membrane. Previous circuits compensated for seal current by means of a variable resistor connected between ground and the input of the current to voltage converter. The purpose of this resistor is to shunt to ground a current equal to the amplified seal current. The disadvantage of this approach is that the actual seal current has a delay imposed on it by the headstage amplifier, whereas the compensation current shunted to ground does not. Therefore, during the onset of a pulse, there is an initial overcompensation for the seal current. The capacitive current transient due to charging of the membrane under the lumen of the pipette is thus seriously distorted, so that integration of this current will not give an accurate measure of membrane area. The degree of this distortion depends on the value of the seal resistance and is difficult to remove accurately using an analog subtraction technique, since the seal resistance can vary considerably between measurements. To solve this problem, we have added circuitry that imposes a time delay on the seal compensation current matching the time delay imposed on the actual seal current by the headstage amplifier. Thus, the capacitive current transient will not 
be distorted by the seal compensation current, and integration of this current transient will give an accurate measure of the total capacitance of the membrane area being recorded.

Before making any measurements of patch capacitance, all stray capacitance was nulled out with the pipette pressed against the bottom of the experimental chamber (coated with Sylgard 184, Dow Corning Corp., Midland, MI), using capacity compensation circuitry that feeds back an amplified and delayed version of the command pulse to the input of the headstage amplifier through a $1 \mathrm{pF}$ capacitor. The majority of the seal current was also removed by the analog subtraction procedure described above. Patch capacitance was then measured by subjecting the patch membrane to a $50 \mathrm{mV}$ hyperpolarizing pulse from resting potential (approximately -50 to $-60 \mathrm{mV}$ for dissociated fibers) and recording the resulting current transient. Pulses were usually $1.75 \mathrm{msec}$ long and were filtered at $5 \mathrm{kHz}$ before input into the patch clamp circuitry in order to decrease the height of the initial capacitive current spike. Measured capacitive currents were filtered at $5 \mathrm{kHz}$ with an eight-pole Bessel filter (Model 902 LPF, Frequency Devices, Inc., Haverhill, MA), digitized at $100 \mathrm{kHz}$ and the average of 16 separate records stored for analysis in a PDP $11 / 73$ computer (Digital Equipment Corp., Marlboro, MA). The voltage command pulse was similarly digitized.

The total effective patch capacitance was determined by averaging the capacitances calculated from integration of the current transients occurring after the on and off of the command pulse, using the formula

$$
\mathrm{C}=\frac{1}{\Delta \mathrm{V}} \int_{0}^{\mathrm{T}}\left(\mathrm{i}_{1}-\mathrm{g}_{\mathrm{u}} \mathrm{V}_{\mathrm{cmd}}\right) \mathrm{dt}
$$

where $i_{t}=$ total measured current (amperes), $\Delta V=$ steady-state magnitude of command pulse (volts), $g_{u}=$ magnitude of any leak or uncompensated seal conductance (calculated from steady-state leakage current occurring during the period $1.38-1.48 \mathrm{msec}$ after the onset of the pulse (Siemens), $V_{c m d}=$ measured command pulse as a function of time (volts), and $\mathrm{T}=$ period 0 to $1 \mathrm{msec}$ after the on or off of the command pulse (sec).

Measurements of $\mathrm{Na}$ currents. Before $\mathrm{Na}$ currents were measured, a steady $50 \mathrm{mV}$ hyperpolarization from rest was applied to the patch membrane for 2 min to activate $\mathrm{Na}$ channels that had been inactivated by a low fiber resting potential. Since the resting potential of dissociated fibers usually varied between -50 and $-60 \mathrm{mV}$, this resulted in a holding potential of -100 to $-110 \mathrm{mV}$ for the patch membrane. Near maximal $\mathrm{Na}$ currents were then elicited by applying a $+100 \mathrm{mV}$ pulse to the patch membrane, depolarizing it to between -10 and $0 \mathrm{mV}$. These values of membrane potential are only approximate, since the loose patch voltage clamp technique does not give a measurement of actual transmembrane potential. This did not represent a serious difficulty for these experiments, since we were only interested in the changes in Na current magnitude caused by bleb formation and not in the absolute voltage dependence of channel properties. However, it was important for our experiments that the magnitude of the actual fiber membrane potential not change appreciably during the course of an experiment, since this would alter Na current magnitude independent of any changes due to bleb formation. Thus, we continuously monitored the time for $\mathrm{Na}$ current to reach peak amplitude. If a change of more than $30 \mu \mathrm{sec}$ in this quantity occurred during an experiment, data from this fiber were not used.

Na channel density is known to vary greatly over the muscle fiber surface, being highly concentrated in the endplate region and sparse near the tendon (Beam et al., 1985; Angelides, 1986; Caldwell et al., 1986; Roberts, 1987; Caldwell and Milton, 1988). In these experiments, Na currents were measured approximately $150 \mu \mathrm{m}$ from the endplate, i.e., at a position where approximately normal midfiber $\mathrm{Na}$ channel densities are to be expected.

When measuring the effects of bleb formation on $\mathrm{Na}$ current, the holding potential was removed and suction was applied (approximately $40 \mathrm{~mm} \mathrm{Hg}$ ) for $5 \mathrm{~min}$ to form a bleb of approximately $25 \mu \mathrm{m}$ in diameter. The holding potential was then reapplied for $2 \mathrm{~min}$, and $\mathrm{Na}$ current was measured. Control experiments were performed using the same protocol but with no suction applied (so that no bleb would form). This was done to ensure that any change in $\mathrm{Na}$ current was purely the result of bleb formation and not due to the measurement procedure.

Sixteen individual $\mathrm{Na}$ current traces for each depolarization were digitized, averaged, and stored in the same manner as described above for the capacity transient currents. Both the command pulse and current records were filtered at $10 \mathrm{kHz}$ instead of $5 \mathrm{kHz}$ so that the kinetics of the currents would not be seriously distorted.
A.
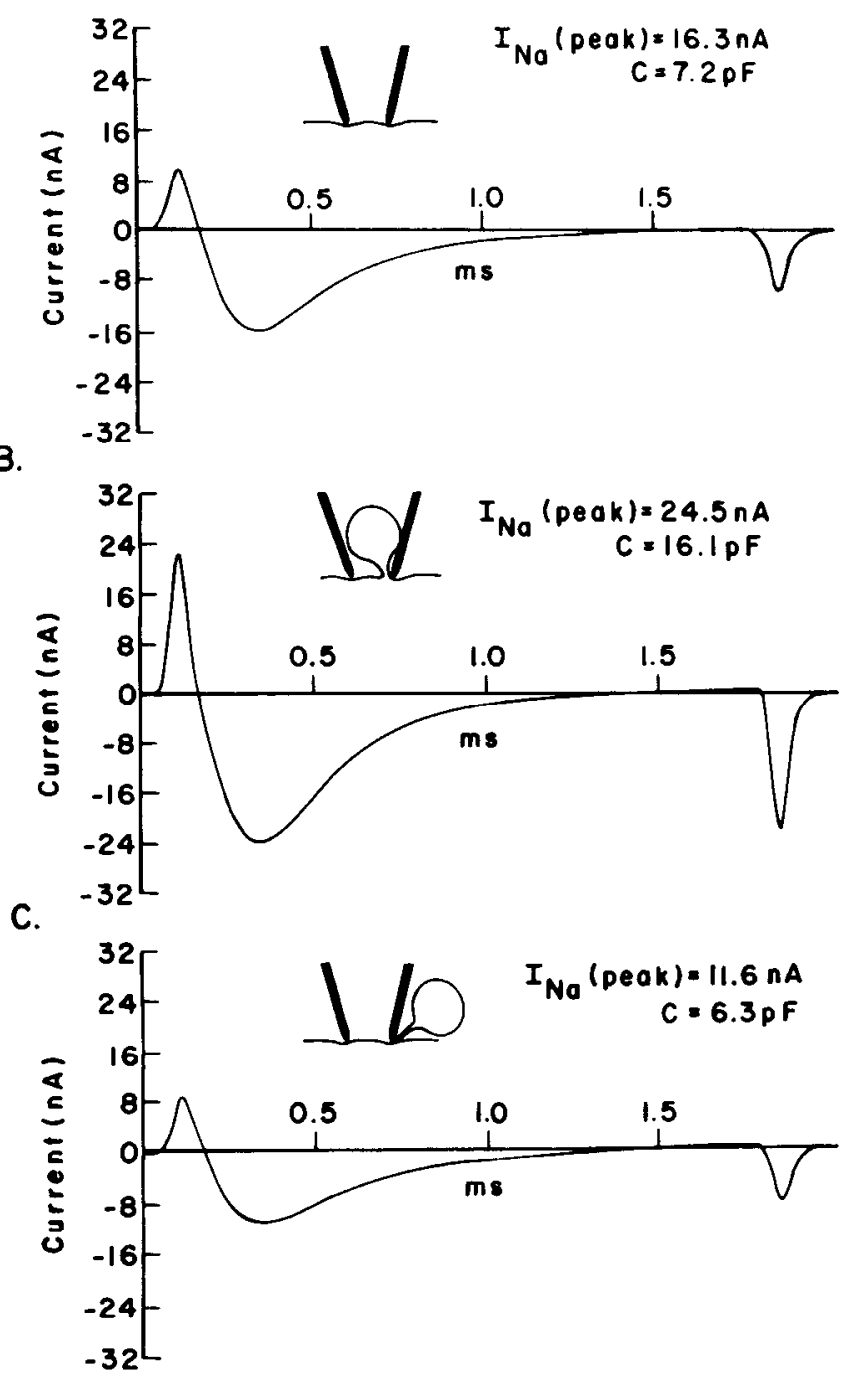

Figure 2. Capacitance and $\mathrm{Na}$ current measurements from the same patch. $A$, Surface membrane without suction and before bleb formation. $B$, Surface membrane with $25 \mu \mathrm{m}$ diameter bleb. $C$, Positive pressure was applied, and the bleb was blown out of the pipette. The bleb often stayed attached to the cell, as shown in the inset.

\section{Results}

\section{Morphology of suction-induced blebs}

Large membrane blebs can be induced to form on the surface of a dissociated mouse skeletal muscle fiber by the application of suction $(10-80 \mathrm{~mm} \mathrm{Hg})$ to the interior of a loose patch pipette pressed against the fiber surface (Fig. 1). These blebs arise suddenly near the inner rim of the pipette, becoming a few micrometers in diameter almost immediately, but they can grow to a diameter of $60 \mu \mathrm{m}$ or more after several minutes. They are not formed by a simple distension of the muscle membrane, since there is no distortion of Z-lines when a bleb forms and enlarges. Very large blebs can have a surface arca of several thousand square micrometers, over 2 orders of magnitude greater than the original area of the patch. From the measurements presented here and elsewhere (R. L. Milton and J. H. Caldwell, unpublished observations), we conclude that these blebs are essentially an aneurysm of the sarcolemma, are formed by a 


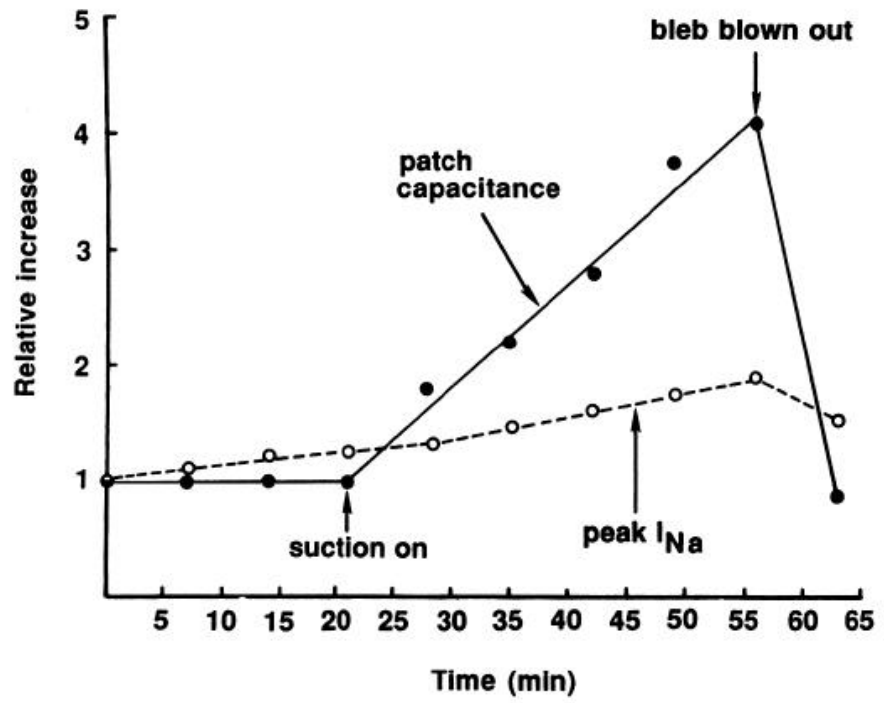

Figure 3. Time course of the change in capacitance and $\mathrm{Na}$ current before and after bleb formation. Bleb growth is indicated by the steady increase in total capacitance. When the bleb surface area was about threefold larger than the patch area, the bleb was blown out of the pipette.

flow of lipid from surrounding surface membrane, and are likely to be free of any attachments to the cytoskeleton.

\section{Na current density in blebs}

Measurements of the peak $\mathrm{Na}$ current that could be elicited from a membrane patch were made both before and after bleb formation, as described in Materials and Methods. Comparison of peak currents before (Fig. $2 A$ ) and after (Fig. $2 B$ ) bleb formation (median bleb diameter $=25 \mu \mathrm{m}$ ) revealed an increase of $37 \pm 23 \%$ (mean $\pm \mathrm{SD} ; n=17$; range $2-76 \%$ ). This value, however, overestimates the effect of bleb formation on Na current, since when the same measurement protocol was followed but suction was not applied (so that a bleb would not form), the second measurement of $\mathrm{Na}$ current resulted in peak values 12 $\pm 8 \%$ (mean $\pm \mathrm{SD} ; n=15$; range $1-46 \%$ ) greater than those obtained from the initial measurement. Thus, the increase in $\mathrm{Na}$ current attributable to bleb formation alone was only $22 \%$ (i.e., $1.37 / 1.12$ ).

The $12 \%$ increase in Na current in the absence of bleb formation was probably due to the increased time of application of the holding potential to the membrane patch. The cause of this time-dependent increase is unknown but may be due to the reversal of a slow inactivation process, as recently described by Almers et al. (1983) and by Simoncini and Stühmer (1987). However, electrophoresis of $\mathrm{Na}$ channels under the pipette rim into the patch may also play a role, since with a $50 \mathrm{mV}$ holding potential, the voltage gradient under the pipette rim may reach values in excess of several hundred volts per centimeter.

Measurements of patch capacitance, made simultaneously with the above measurements of $\mathrm{Na}$ current, showed an increase of $147 \pm 44 \%$ (mean \pm SD; $n=17$; range $78-218 \%$ ); i.e., the final capacitance was almost 2.5 times that without the bleb. Thus, the new membrane drawn into the pipette by suction was severely deficient in $\mathrm{Na}$ channels, since a $147 \%$ increase in membrane area resulted in only a $22 \%$ increase in Na current. This would indicate a $\mathrm{Na}$ channel density in bleb membrane only about $15 \%$ that of the membrane originally under the pipette.

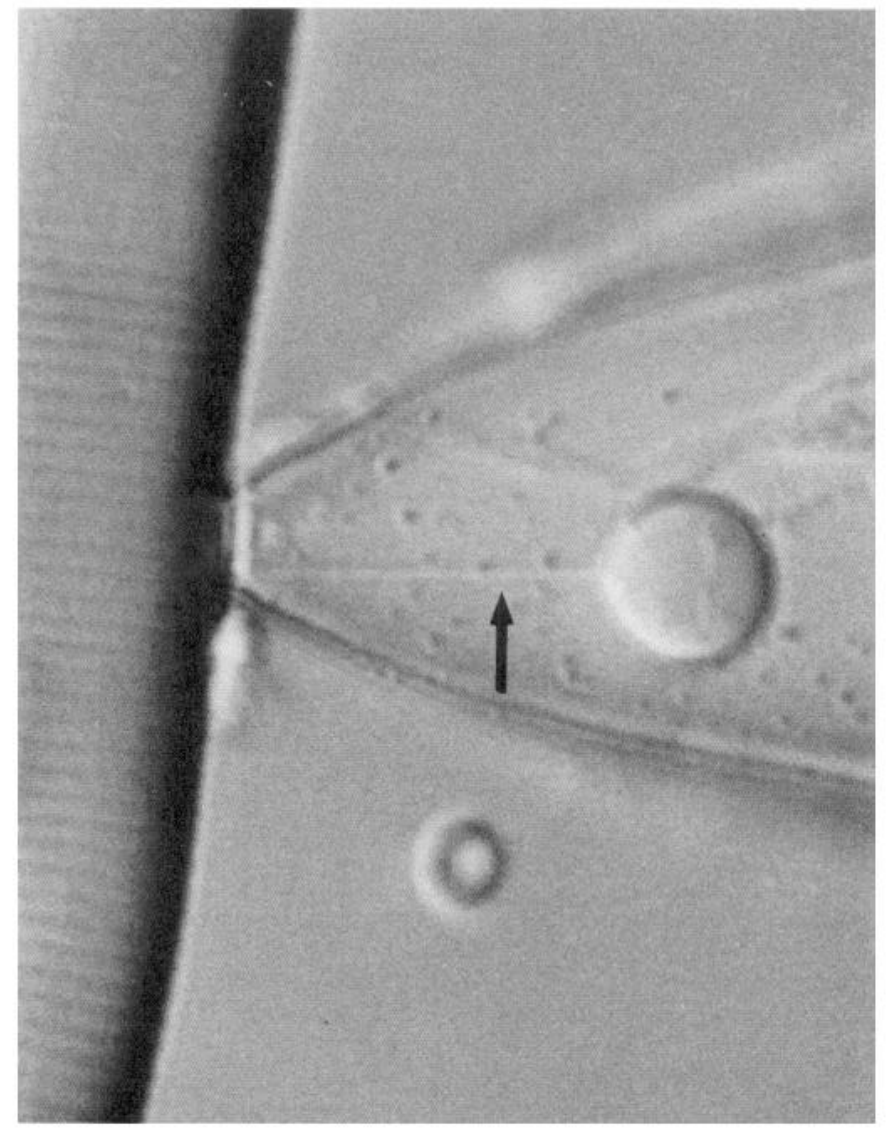

Figure 4. A long tether (arrow) connects the bleb to the cell. Length of the tether is $30 \mu \mathrm{m}$.

We show in the next section that this is an underestimate of the channel density in the bleb.

The data plotted in Figure 3 were obtained with a different protocol. In this fiber, a $50 \mathrm{mV}$ hold was applied for $2 \mathrm{~min}$, and peak $\mathrm{Na}$ current was measured. The hold was then removed for $5 \mathrm{~min}$ and reapplied for $2 \mathrm{~min}$, and $\mathrm{Na}$ current was remeasured. This procedure was repeated 3 times, and suction was then applied to produce a bleb. The sequence of removing the hold for $5 \mathrm{~min}$, reapplying it for $2 \mathrm{~min}$, and measuring peak $\mathrm{Na}$ currents (along with patch capacitance) was repeated 5 times. As can be seen from Figure 3, peak Na current slowly increased with each subsequent measurement before bleb formation at an average rate of about $1 \%$ per minute. Following bleb formation, this rate increased to about $2 \%$ per minute. However, the patch capacitance during this period increased at a rate of approximately $8 \%$ per minute, again indicating that new membrane drawn into the pipette by suction has a reduced $\mathrm{Na}$ channel density.

Although we made no detailed study of $\mathrm{Na}$ current kinetics before and after bleb formation, we noticed no changes in channel kinetics after bleb formation. No inflections in the current records were ever present that would indicate that the currents were arising from 2 separate channel populations with differing kinetics. When the peak current of Figure $2 A$ (before the bleb) was scaled up to the peak current in Figure $2 B$ (with a bleb present) and the currents were plotted together, the 2 current traces were indistinguishable. This suggests that $\mathrm{Na}$ channel behavior is similar in bleb and normal surface membrane. In 
some cases, long, thin tethers held the bleb to the surface (Fig. 4). For a few of these, there was an abrupt slowing of kinetics and a decrease in the peak current. We assume that this change in kinetics was due to an increase in the series resistance through the tether. In 2 blebs, the kinetics alternated between normal and slow, and the switch from a slowed to normal kinetics coincided with a bolus of fluid (cytoplasm) moving up the tether into the bleb. These burps evidently opened the tether lumen temporarily because $\mathrm{Na}$ currents had normal kinetics for a few seconds after each burp.

\section{Redistribution of $\mathrm{Na}$ channels}

We initially assumed that bleb membrane contained only the additional channels drawn into the pipette during suction. We tested this assumption and found that some of the Na channels already under the pipette had also moved up into the bleb. To determine to what extent such a redistribution had occurred, measurements of peak $\mathrm{Na}$ current were made with the bleb inside the pipette. The pipette was then raised slightly (about 2 $\mu \mathrm{m}$ ), positive pressure was applied to the lumen of the pipette to blow the bleb out, the pipette was then lowered, and peak $\mathrm{Na}$ current remeasured (Fig. 2C). Since these manipulations should have no direct effect on Na current, any change in current after bleb removal could be attributed to the loss of $\mathrm{Na}$ channels in the bleb. The results of these measurements revealed a drop in membrane capacitance of $59 \pm 11 \%$ (mean $\pm \mathrm{SD} ; n=13$; range $38-80 \%$ ) after blowing the bleb out of the pipette. This is in close agreement with the $147 \%$ increase seen with bleb formation (since $1.47 / 2.47=59.5 \%$ ). Given the high mobility of membrane lipids and the return of capacitance to its original (pre-bleb) value, we conclude that lipids that moved from the patch into the bleb were replaced by lipids from surrounding membrane.

Peak Na current was found to decrease by $43 \pm 17 \%$ (mean $\pm \mathrm{SD} ; n=13$; range $3-65 \%$ ), more than twice what would have been predicted $(0.22 / 1.22=18 \%)$ on the basis of the increase in current after bleb formation. Comparison of the peak $\mathrm{Na}$ currents that could be elicited from the patch membrane before bleb formation and after bleb removal revealed a concomitant drop in patch Na current of $29 \pm 22 \%$ (mean $\pm \mathrm{SD} ; n=13$; range $6-62 \%$ ). These results, therefore, imply that a redistribution of $\mathrm{Na}$ channels had occurred: some channels under the pipette moved into bleb membrane. This suggests that the source of the Na channels in bleb membrane is the local membrane surrounding the site of bleb formation. Since blebs form at the inner pipette rim, this local membrane includes both a region under the pipette and a region outside the pipette (Fig. 5). Only that region outside the pipette (hatched region in Fig. $5 b$ ) contributes to the $22 \%$ overall increase in $\mathrm{Na}$ current seen after bleb formation.

The above data can also be used to determine how Na channel density in bleb membrane compares with that of patch membrane before bleb formation. Average peak Na current density of the patch, measured by dividing peak Na current by patch capacitance, was found to be $2.10 \pm 1.09 \mathrm{nA} / \mathrm{pF}$ (mean $\pm \mathrm{SD}$; $n=13$; range $1.18-5.33$ ). Calculation of the peak $\mathrm{Na}$ current density in bleb membrane, made by dividing the change in $\mathrm{Na}$ current after bleb removal by the concurrent change in patch capacitance, revealed a value of $0.91 \pm 0.56 \mathrm{nA} / \mathrm{pF}$ (mean \pm $\mathrm{SD} ; n=13$; range $0.11-2.45$ ). When these current and capacitance values were compared for individual patches rather than first averaging, peak $\mathrm{Na}$ current density in bleb membrane was
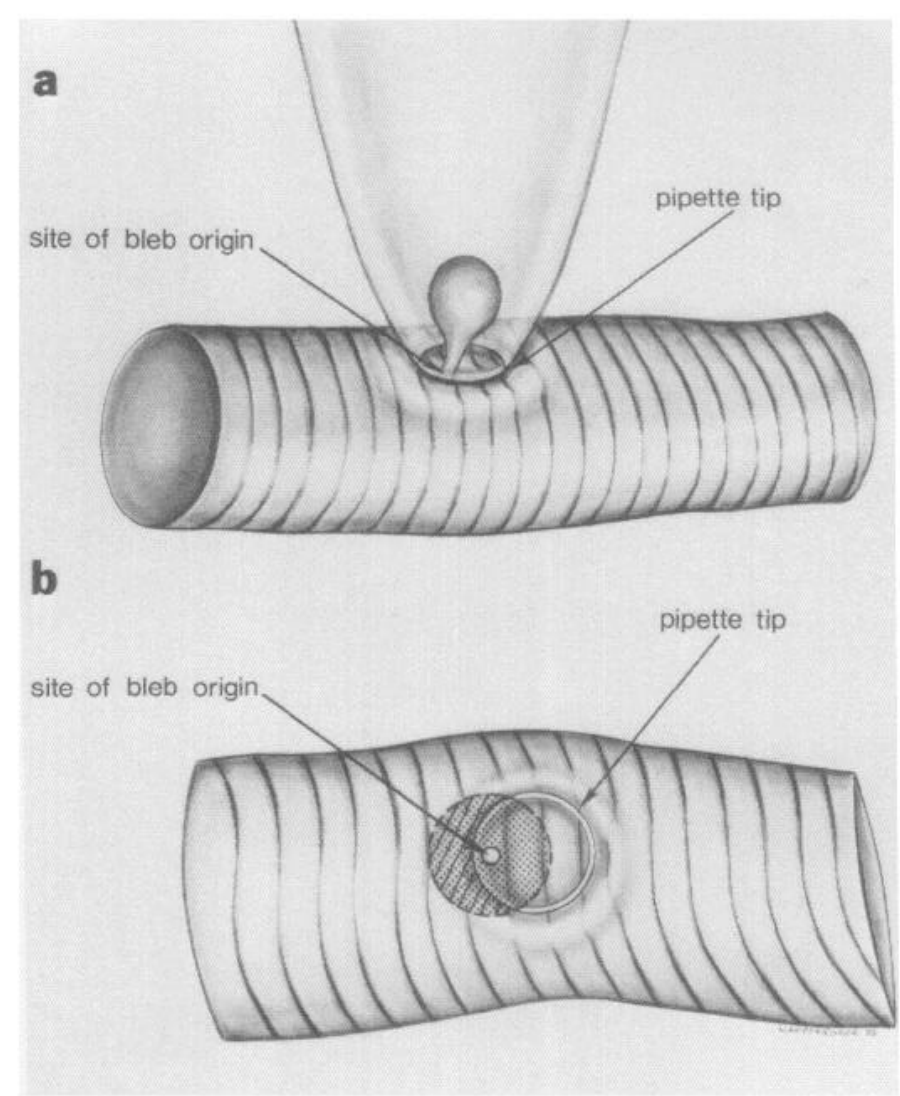

Figure 5. a, Schematic view of pipette to combine experimental observations with the pipette either parallel or perpendicular to the bottom of the chamber. The bleb origin is at the inner rim of the pipette. $b$, Vertical view of pipette tip and bleb origin. The stippled circular area around the origin contributes $\mathrm{Na}$ channels to the bleb. Channels from the hatched area (outside the pipette) cause the increase $(22 \%)$ in $\mathrm{Na}$ current, whereas the shift of channels from surface membrane inside the pipette is only apparent when the bleb is blown out.

on average $47 \pm 20 \%$ (mean $\pm \mathrm{SD} ; n=13$; range $4-71 \%$ ) of the peak Na current density measured from the membrane patch before bleb formation. Thus, bleb membrane is not representative of normal surface membrane but is partially depleted of $\mathrm{Na}$ channels.

\section{$\mathrm{Na}$ current from isolated blebs}

To verify unequivocally that the drop in Na current seen after bleb removal was due to $\mathrm{Na}$ channels in bleb membrane, we measured Na currents in blebs that had been completely pulled free from any attachment to the muscle fiber (Fig. 6A). These experiments were difficult to perform, since the pressure within the pipette must be very finely controlled. Too much pressure will blow the bleb out of the pipette, and too little pressure will result in low seal resistance. We were on 4 occasions able to keep an isolated bleb in the pipette long enough to apply a holding potential of about $-100 \mathrm{mV}$ for $2 \mathrm{~min}$ and to measure peak Na current and membrane capacitance (Fig. $6 b$ ). The polarity of the hold and direction of current are explained by the equivalent circuit in Fig. $6 C$. These measurements revealed a peak Na current density of $0.94 \pm 0.34 \mathrm{nA} / \mathrm{pF}$ (mean $\pm \mathrm{SD} ; n$ $=4$; range $0.50-1.21$ ). This average is reasonably close to the value of $0.91 \mathrm{nA} / \mathrm{pF}$ obtained from the bleb removal data, but it probably underestimates the actual $\mathrm{Na}$ channel density in 

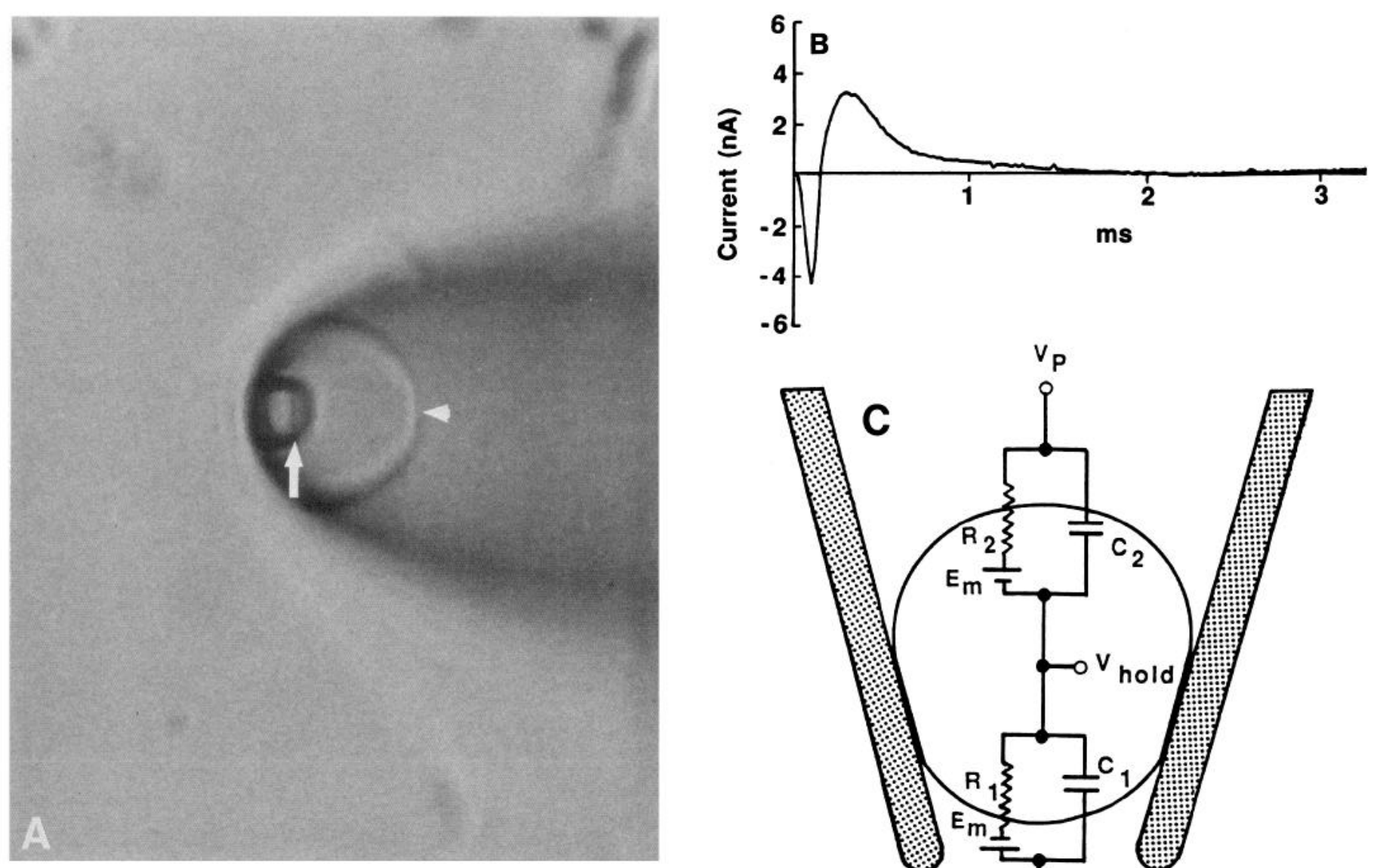

Figure 6. A, An isolated bleb. A vertical view of the pipette tip, rather than the horizontal view shown in Figure 1. The dark circle is the pipette opening (arrow), and the larger circle (arrowhead) is the bleb, which was $37 \mu \mathrm{m}$ in diameter. B, Capacitive and Na current from an isolated bleb. The polarity of the holding potential and the test potential had to be reversed to generate Na current. The early negative current is the capacitive transient. The Na current is outward because it was generated only by the vesicle membrane closest to the tip $\left(R_{1}\right.$ and $\left.C_{1}\right)$; $120 \mathrm{mV}$ depolarizing pulse imposed on a holding potential of approximately $-125 \mathrm{mV}$. $C$, Equivalent circuit of the vesicle to account for the current in $B$. The vesicle acts as a voltage divider. The proximal membrane $\left(R_{2}\right.$ and $\left.C_{2}\right)$ was about sixfold greater in area than the distal $\left(R_{1}, C_{1}\right)$ membrane. Thus, the majority of the holding potential and test potential was applied to the distal membrane. This membrane is reversed in orientation; thus, the Na currents also will be reversed in direction. $C_{1}+C_{2}$ measured before bleb removal. $C_{1} C_{2} /\left(C_{1}+C_{2}\right)$ measured after bleb removal. $C_{1}=3$ pF, $C_{2}=$ $18 \mathrm{pF}$; hence $\mathrm{R}_{1}=6 \mathrm{R}_{2}$.

these isolated blebs because the amount of vesicle membrane is not sufficient to maintain the interior of the bleb at constant potential during the peak values of the $\mathrm{Na}$ currents. Hence, these results are presented more for their unequivocal demonstration of the existence of $\mathrm{Na}$ channels in bleb membrane than for their quantitative usefulness.

\section{Discussion}

Application of suction to the back of a loose patch voltage clamp recording pipette can induce formation of membrane blebs inside the pipette. Measurements of voltage-gated $\mathrm{Na}$ current with and without these blebs have led to the following conclusions. First, measurements of membrane current density using the loose patch clamp technique will be inaccurate if membrane blebs occur. Second, a significant fraction of $\mathrm{Na}$ channels in the extrajunctional region of adult mouse muscle may be mobile. We describe a simple model in the Appendix to reconcile the steep gradients of $\mathrm{Na}$ channel density found in these fibers with the existence of a large fraction of mobile channels.

\section{Current density}

During the formation of an electrical seal for loose patch clamp recording, suction is often applied to the back of the pipette to increase the seal resistance at the rim of the tip. This procedure works well on intact tissue. With enzymatically dissociated cells or tissue cultured cells, however, blebs often appeared inside the pipette during suction. We expected to find either very few or no Na channels in these blebs, since the results of Stühmer and Almers (1982) and the large gradients of channel density at the endplate and tendon suggested that all $\mathrm{Na}$ channels are immobile. However, the blebs were not devoid of channels; $\mathrm{Na}$ channel density was almost $50 \%$ that of normal sarcolemma. Thus, bleb formation can introduce variability into measurements of current density. This variability will be a function of the size of the vesicle and the mobility of the channels being studied. There is no simple way to correct for this variability. If current is measured without normalizing to membrane area, the calculated current density will be too high because of ad- 
ditional channels drawn into the pipette. If current is normalized to membrane area (using measured capacitance), calculated density for normal surface membrane will be too low because the bleb membrane is protein poor. In previous experiments mapping Na current density (Beam et al., 1985; Caldwell et al., 1986; Caldwell and Milton, 1988), we excluded measurements when blebs appeared. Similar blebs have been observed by Roberts and Hudspeth (1987) when recording from dissociated hair cells.

\section{Bleb membrane}

What is the source and composition of the bleb membrane? A priori blebs could be (1) native membrane, i.e., an extension of normal sarcolemma, (2) lipid flowing from sarcolemma and transverse tubules, carrying with it mobile proteins, or (3) primarily lipid and mobile proteins but also comprised of patches of native membrane.

The following observations make it extremcly unlikcly that the bleb is composed of native membrane identical to the sarcolemma. (1) Na channel density is much lower than that of the sarcolemma. (2) Carbon particles and latex beads on the muscle surface do not flow into the pipette when blebs form, which implies that the extracellular matrix does not move. Thus, any proteins linked to the extracellular matrix would probably not move into the bleb. (3) The myofilament matrix (Z-lines and A-I bands) is unaltered even by the formation of large blebs. Membrane proteins attached to this matrix would have to be detached in order to move into the bleb because no intact cytoskeletal matrix is seen in electron micrographs of these blebs (unpublished observations). This detachment is energetically unlikely.

Although we are unable to provide data to distinguish between the second and third possibilities, it is unlikely that patches of membrane or even single proteins are broken loose from cytoskeletal or extracellular attachments by bleb formation. This conclusion is based on a comparison of the kinetic energy involved in bleb formation with the thermal energy that dominates lipid and protein movement. If we assume that a $5 \mu \mathrm{m}$ diameter bleb formed in $0.1 \mathrm{sec}$ with a tether $1 \mu \mathrm{m}$ in diameter, the maximum velocity of lipid at the tether is about $100 \mu \mathrm{m} /$ sec. Assuming that the molecular weight of a typical lipid molecule is $1000 \mathrm{Da}$, the molecule's maximum kinetic energy $(1 / 2$ $\mathrm{mv}^{2}$ ) will be about $10^{-32} \mathrm{~J}$. At room temperature $\left(300^{\circ} \mathrm{K}\right)$, thermal energy (kT) is about $4 \times 10^{-21} \mathrm{~J} /$ molecule. Thus, the additional kinetic energy imparted to a lipid molecule as a result of bleb formation is about $10^{12}$ times smaller than its thermal energy, and the forces imposed on membrane proteins by lipid flow will be insignificant compared to the forces the protein normally experiences due to thermal energy. However, even if local forces at or very near to the site of bleb formation are higher than these estimates and are possibly disruptive enough to rip Na channels free of their binding sites, this is still unlikely to be responsible for most of the Na channels in bleb membrane. This conclusion arises from the fact that blebs originate from small regions of surface membrane that would not contain sufficient numbers of $\mathrm{Na}$ channels to account for the densities found in blebs. Thus, most $\mathrm{Na}$ channels in bleb membrane must be recruited from sarcolemma surrounding the site of bleb formation. This membrane is not significantly disturbed during bleb formation or growth, and thus $\mathrm{Na}$ channels in this region are unlikely to be torn free of their binding sites. Also, if channels in bleb membrane are provided by pulling channels loose from cytoskeletal binding sites, these channels should diffuse back out of the bleb and rebind after bleb formation.

\section{Na channel mobility}

If bleb formation does not break channel attachments to the cytoskeleton, how do we interpret the existence of $\mathrm{Na}$ channels in the bleb? It is possible that bulk flow of lipid during bleb formation pulled in channels that had low mobility but were not attached directly to the cytoskeleton. Evidence that the cytoplasmic tails of some membrane proteins can be almost totally removed without altering mobility (Edidin and Zuniga, 1984; Livneh et al., 1986; Scullion et al., 1987) strongly suggests that binding to cytoskeletal elements may not be the only means of reducing membrane protein mobility. How might channel movement be constrained other than by bonds to the cytoskeleton (or, equivalently, the extracellular matrix)? Low mobility could be a result of steric hindrance, for example, if cytoplasmic or extracellular channel regions bump into structural proteins. Steric hindrance effects have been modeled by Koppel et al. (1981). Another way to affect mobility is to alter local membrane viscosity, perhaps by having lipid domains (Yechiel and Edidin, 1987). The $\mathrm{Na}$ channel is heavily fatty acylated (Levinson et al., 1986) and might create its own lipid environment. Selfaggregation is another possible cause of reduced mobility. Aggregation of membrane proteins into clusters or rafts will slow diffusion and reduce the effective concentration of free channels (Saffman and Delbruck, 1975). Bleb formation could modify or disrupt these constraints.

A second explanation, which is explored in the remainder of the discussion, is that the increase in peak $\mathrm{Na}$ current with bleb formation is not an artifact due to disruption of constraints but rather implies that at least some of the $\mathrm{Na}$ channels in normal surface membrane are mobile. If we assume a simple model that divides membrane proteins into distinct mobile and immobile fractions, it is reasonable to suppose that at equilibrium the $\mathrm{Na}$ channel density in bleb membrane should be equal to the density of mobile channels in the adjoining normal surface membrane. Thus, our results would imply that at least $50 \%$ of the $\mathrm{Na}$ channels in normal surface membrane are mobile (at a location approximately $150 \mu \mathrm{m}$ from the endplate). The actual percentage of mobile channels may be even higher than $50 \%$, since the fact that peak $\mathrm{Na}$ currents measured in patch membrane after bleb removal were $29 \%$ lower than the initial measurement made before bleb formation indicates that $\mathrm{Na}$ channels in adjacent surface membrane had not yet diffused into the patch to replenish those channels lost to the bleb. Thus, diffusional equilibrium had not yet been achieved. This slow approach to equilibrium is not surprising given the low $\mathrm{Na}$ channel diffusion coefficients measured in adult mouse skeletal muscle $\left[\mathrm{D}=3 \times 10^{-10} \mathrm{~cm}^{2} / \mathrm{sec}\right.$ (Angelides, 1986) implies that a $\mathrm{Na}$ channel would take about $1 \mathrm{hr}$ to diffuse $20 \mu \mathrm{m}$ ].

Interpreted in this way, our results are in substantial agreement with those of Angelides (1986) who found, using fluorescence photobleaching recovery techniques, that at $200 \mu \mathrm{m}$ from the endplate in adult mouse diaphragm muscle, approximately $55 \%$ of the $\mathrm{Na}$ channels are mobile. However, these results are in disagreement with those of Stühmer and Almcrs (1982), who, using ultraviolet irradiation and loose patch voltage clamping, found $\mathrm{Na}$ channels in frog sarcolemma to be virtually immobile. Although this disagreement may result from a species difference (see below), previous measurements (Caldwell et al., 1986; Caldwell and Milton, 1988) of the distribution of Na channels near 


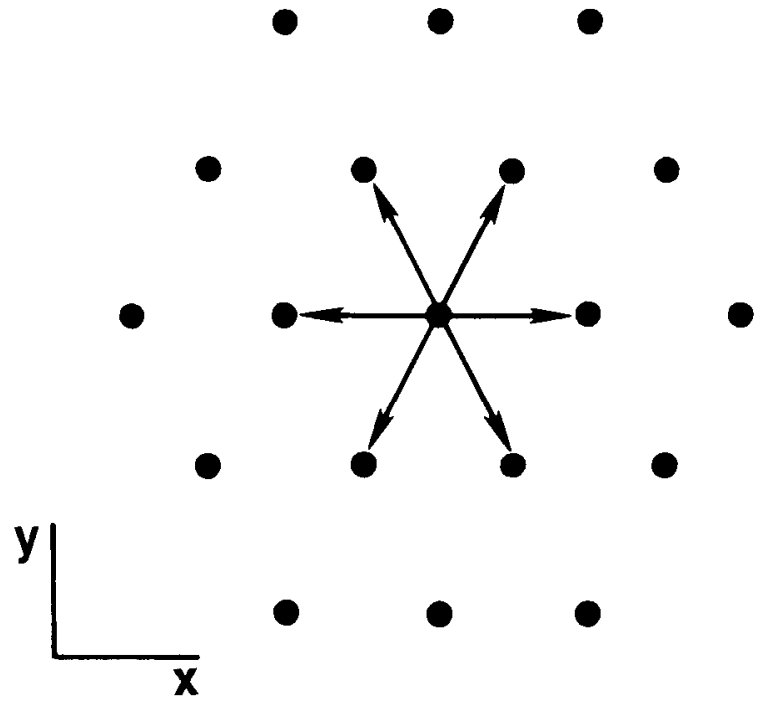

Figure 7. Diagram of the binding sites that are assumed to be equally spaced in order to simplify the derivation of equation 1.

the tendon in mouse, rat, and snake muscle also seem inconsistent with a high $\mathrm{Na}$ channel mobility. These measurements revealed that $\mathrm{Na}$ channel density begins to decrease at about $500 \mu \mathrm{m}$ from the tendon, falling to less than $10 \%$ of its midfiber value by about $50 \mu \mathrm{m}$ from the fiber end. These results would seem to imply a low mobility for $\mathrm{Na}$ channels, unless some continuous process of channel redistribution is taking place or barriers for longitudinal diffusion exist along the muscle fiber. Ihe following section and the Appendix present a model that reconciles these apparent inconsistencies without invoking either redistribution or barriers.

\section{Binding site model}

The apparent contradiction between a steep spatial gradient and a large mobile fraction may stem from the use of an oversimplified model, which divides $\mathrm{Na}$ channels into distinct and separate mobile and immobile classes. An alternative, which is developed in the Appendix, is the binding site model. If $\mathrm{Na}$ channels tended to cluster around specific binding sites in the sarcolcmma, a nonuniform distribution of channcls would simply be a consequence of the heterogeneous distribution of these sites. Similar clustering sites might be responsible for the micrometer-scale domains observed by Yechiel and Edidin (1987). The recent finding of Srinivasan et al. (1988) that the cytoskeletal proteins ankyrin and spectrin copurify with $\mathrm{Na}$ channels and the identification of a $33 \mathrm{kDa}$ cytoskeletal protein with a high affinity for the $\mathrm{Na}$ channel by Edelstein et al. (1988) lend credence to the binding site model. Additionally, if peripheral channels in these clusters were not tightly bound, exchange of channels between clusters could occur. These loosely bound channels would appear in mobility measurements as a mobile fraction with a diffusion coefficient much lower than that expected from bilayer viscosity. Under these circumstances, measured diffusion coefficients would tend to vary inversely with the density of aggregation sites (Appendix, equation 8) since channels in regions of high site density would spend proportionately more time bound in clusters than would channels in regions of low site density. Just such a variation of diffusion coefficients with distance from the endplate (and also, therefore, with channel density) was found for adult mouse muscle (Angelides, 1986). An inverse relation between channel density and diffusion coefficient was also measured in the soma and axon hillock of neurons (Angelides et al., 1988). As additional support, Yechiel and Edidin (1987) found an inverse relationship between fluorescence intensity (and, therefore, presumably density) and the diffusion coefficient of labeled membrane proteins in human fibroblasts.

In addition to predicting an inverse relationship between channel density and the measured diffusion coefficient, the binding site model demonstrates that large gradients in channcl density can exist without requiring that all the channels be permanently immobile or have their mobility restricted by barriers. This is a result of the fact that channels in regions of high binding site density will spend proportionately more time bound than will channels in regions of lower binding site density. This increased bound time compensates for the higher channel density, so that the actual number of channels unbound at any one instant in time (free channel density) is independent of binding site density (and, hence, the total channel density as well). Therefore, a gradient in channel density can exist without a constant flux of channels from the high to low density regions.

Although our results may appear to be in disagreement with those of Stühmer and Almers (1982), this is not necessarily the case. Since small variations in the binding affinity or in the number of binding sites as compared to the number of channels might greatly affect measured mobile fractions and diffusion coefficients, this could be the basis of the different channel mobilities observed in mouse and frog.

\section{Appendix}

We assume a simple idealized 2-dimensional random walk of a channel between equally spaced binding sites. With each step, the channel moves a distance $\lambda$ with equal probability in any one of the 6 directions shown (Fig. 7). If we denote the channel's displacement from its initial position in the $x$-direction as $x_{n}$, on the next step there is a probability of one third that

$$
\mathrm{x}_{\mathrm{n}+1}=\mathrm{x}_{\mathrm{n}} \pm \lambda
$$

and a probability of two thirds that

$$
x_{n+1}=x_{n} \pm \lambda / 2
$$

Similarly, for displacement in the $y$-direction, we will have

$$
\mathrm{y}_{\mathrm{t}+1}=\mathrm{y}_{\mathrm{u}}
$$

with a probability of one third, and

$$
\mathrm{y}_{\mathrm{n}+1}=\mathrm{y}_{\mathrm{n}} \pm \frac{\sqrt{3} \lambda}{2}
$$

with a probability of two thirds. Thus the mean square displacement in the $\mathrm{x}$-direction after $\mathbf{n}+1$ steps, $\left\langle\mathrm{x}^{2}{ }_{\mathrm{n}+1}\right\rangle$, is given by

$$
\left\langle\mathbf{X}_{n+1}^{2}\right\rangle=\frac{1}{3}\left(\left\langle\mathbf{X}_{n}{ }_{n}\right\rangle+\lambda^{2}\right)+\frac{2}{3}\left(\left\langle\mathbf{X}_{n}^{2}\right\rangle+\frac{\lambda^{2}}{4}\right)=\left\langle\mathrm{X}_{n}^{2}\right\rangle+\frac{\lambda^{2}}{2}
$$

Similarly,

$$
\left\langle\mathbf{y}^{2}{ }_{n+1}\right\rangle=\frac{1}{3}\left\langle y_{n}^{3}\right\rangle+\frac{2}{3}\left(\left\langle y_{n}^{3}\right\rangle+\frac{3 \lambda^{2}}{4}\right)=\left\langle y_{n}^{2}\right\rangle+\frac{\lambda^{2}}{2}
$$

Hence, the mean square displacement of the channel after $n+1$ steps is

but since

$$
\left\langle r_{n+1}^{2}\right\rangle-\left\langle r_{n}^{2}\right\rangle+\lambda^{2}
$$

$$
\left\langle\mathbf{r}^{2}{ }_{1}\right\rangle=\lambda^{2}
$$


we have

$$
\left\langle\mathrm{r}_{\mathrm{n}}^{2}\right\rangle=\mathrm{n} \lambda^{2}
$$

In two dimensions we also have

$$
\left\langle\mathrm{r}^{2}\right\rangle=4 \mathrm{D}_{\mathrm{eff}} \mathrm{t}
$$

where $\left\langle\mathbf{r}^{2}\right\rangle=$ mean displacement of the diffusing particle from its initial position $(\mathrm{cm}), \mathrm{t}=$ time $(\mathrm{sec})$, and $\mathrm{D}_{\text {eff }}=$ effective diffusion coefficient $\left(\mathrm{cm}^{2} / \mathrm{sec}\right)$.

Thus, from equations 1 and 2 ,

$$
\mathrm{D}_{\mathrm{eff}}=\frac{\lambda^{2}}{4 \tau}
$$

where $\tau=\mathrm{t} / \mathrm{n}=$ time for channel to make one step (sec). We assume that the time necessary to make one step, $\tau$, consists of 2 components, the actual time spent freely diffusing, $\tau_{f}$, and the time spent bound to a binding site before the step, $\tau_{\mathrm{b}}$, i.e.,

$$
\tau=\tau_{\mathrm{f}}+\tau_{\mathrm{b}}
$$

Now again in 2 dimensions, we have

$$
\tau_{\mathrm{f}}=\frac{\lambda^{2}}{4 \mathrm{D}}
$$

where $\mathrm{D}=$ the diffusion coefficient of the mobile (unhindered) channel in the lipid bilayer $\left(\mathrm{cm}^{2} / \mathrm{sec}\right)$. From equations 3,4 , and 5

$$
\mathrm{D}_{\mathrm{eff}}=\frac{\tau_{\mathrm{f}}}{\tau_{\mathrm{f}}+\tau_{\mathrm{b}}} \mathrm{D}
$$

Thus, channel binding lowers the apparent diffusion coefficient by a factor

$$
\tau_{\mathrm{f}} /\left(\tau_{\mathrm{f}}+\tau_{\mathrm{b}}\right)
$$

Additionally, the average density of channels not bound to a site at any instant of time, i.e., the free channel density, $\sigma_{\mathfrak{f}}$, is given by

$$
\sigma_{\mathrm{f}}=\frac{\tau_{\mathrm{f}}}{\tau_{\mathrm{f}}+\tau_{\mathrm{b}}} \sigma_{\mathrm{t}}
$$

where $\sigma_{\mathrm{t}}=$ the total channel density. Note that the free channel density as defined here is not equivalent to the mobile fraction measured with the fluorescence recovery after photobleaching technique. From equations 6 and 7 we have

$$
\mathrm{D}_{\mathrm{eff}}=\frac{\sigma_{\mathrm{f}} \mathrm{D}}{\sigma_{\mathrm{t}}}
$$

At equilibrium, if there are no barriers to diffusion, $\sigma_{\mathrm{f}} \mathrm{D}$ should be constant along the length of the fiber. $D_{\text {eff }}$ is then proportional to $1 / \sigma$.

The above simple model assumes that when a $\mathrm{Na}$ channel diffuses to a binding site, the probability of binding is close to one. This assumption will be valid if (a) the binding of channels to sites is one to one and there are many morc binding sites than channcls, or (b) Na channels tend to cluster around binding sites so that many channels can be associated with any one site. Also, under these conditions, the average time a channel stays bound to an individual binding site, $\tau_{b}$, will depend on temperature and binding affinity only and will not be a function of binding site density. We also assume that properties of binding sites are the same over the length of the fiber. Therefore, since

$$
\sigma_{\mathrm{f}}=\left(\tau_{\mathrm{f}} / \tau_{\mathrm{b}}\right) \sigma_{\mathrm{b}}
$$

where $\sigma_{\mathrm{b}}$ (the density of bound channels) is proportional to $1 / \lambda^{2}$ and $\tau_{\mathrm{f}}$ is proportional to $\lambda^{2}$, the free channel density will be independent of the binding site density. Thus, regions of high channel density (due to a high density of binding sites) can exist adjacent to regions of low channel density (due to a low density of binding sites) without having a diffusional gradient for channel movement, even if the channels are not tightly bound to their binding sites and have a high measured mobile fraction.

\section{References}

Almers, W., and C. Stirling (1984) Distribution of transport proteins over animal cell membranes. J. Membr. Biol. 77: 169-186.
Almers, W., P. R. Stanfield, and W. Stühmer (1983) Slow changes in currents through sodium channels in frog muscle membrane. J. Physiol. 339: 253-271.

Angelides, K. J. (1986) Fluorescently labelled $\mathrm{Na}^{+}$channels are localized and immobilized to synapses of innervated muscle fibers. Nature 321: 63-66.

Angelides, K. J., L. W. Elmer, D. Loftus, and E. Elson (1988) Distribution and lateral mobility of voltage-dependent sodium channels in neurons. J. Cell Biol. 106: 1911-1925.

Axelrod, D. (1983) Lateral motion of membrane proteins and biological function. J. Membr. Biol. 75: 1-10.

Beam, K. G., J. H. Caldwell, and D. T. Campbell (1985) Na channels in skeletal muscle concentrated near the neuromuscular junction. Nature 313: 588-590.

Bekoff, A., and W. J. Betz (1977) Physiological properties of dissociated muscle fibers obtained from innervated and denervated adult rat muscle. J. Physiol. (Lond.) 271: 25-40.

Caldwell, J. H., and R. L. Milton (1988) Sodium channel distribution in normal and denervated rodent and snake skeletal muscle. J. Physiol. (Lond.) 401: 145-161.

Caldwell, J. H., D. T. Campbell, and K. G. Beam (1986) Na channel distribution in vertebrate skeletal muscle. J. Gen. Physiol. 87: 907932.

Edelstein, N. G., W. A. Catterall, and R. T. Moon (1988) Identification of a 33-kilodalton cytoskeletal protein with high affinity for the sodium channel. Biochemistry 27: 1818-1822.

Edidin, M., and M. Zuniga (1984) Lateral diffusion of wild-type and mutant $\mathrm{L}^{\mathrm{d}}$ antigens in L cells. J. Cell Biol. 99: 2333-2335.

Koppel, D. E., M. P. Sheetz, and M. Schindler (1981) Matrix control of protein diffusion in biological membranes. Proc. Natl. Acad. Sci. USA 78: 3576-3580.

Levinson, S. R., D. S. Duch, B. W. Urban, and E. Recio-Pinto (1986) The sodium channel from Electrophorus electricus. Ann. NY Acad. Sci. 479: 162-178.

Livneh, E., M. Benveniste, R. Prywes, S. Felder, Z. Kam, and J. Schlessinger (1986) Large deletions in the cytoplasmic kinase domain of the epidermal growth factor receptor do not affect its lateral mobility. J. Cell Biol. 103: 327-331.

Roberts, W. M. (1987) Sodium channels near endplates and nuclei of snake skeletal muscle. J. Physiol. 388: 213-232.

Roberts, W. M., and A. J. Hudspeth (1987) Co-localization of Ca channels with Ca-activated $\mathrm{K}$ channels in hair cells of the bullfrog's sacculus. Soc. Neurosci. Abstr. 13: 177.

Saffman, P. G., and M. Delbruck (1975) Brownian motion in biological membranes. Proc. Natl. Acad. Sci. USA 72: 3111-3113.

Scott, R. E. (1976) Plasma membrane vesiculation: A new technique for isolation of plasma membrancs. Science 194: 743-745.

Scullion, B. F., Y. Hou, L. Puddington, J. K. Rose, and K. Jacobson (1987) Effects of mutations in three domains of the vesicular stomatitis viral glycoprotein on its lateral diffusion in the plasma membrane. J. Cell Biol. 105: 69-75.

Simoncini, L., and W. Stühmer (1987) Slow sodium channel inactivation in rat fast-twitch muscle. J. Physiol. (Lond.) 383: 327-337.

Srinivasan, Y., L. Elmer, J. Davis, V. Bennett, and K. Angelides (1988) Ankyrin and spectrin associate with voltage-dependent sodium channels in brain. Nature 333: 177-180.

Standen, N. B., P. R. Stanfield, T. A. Ward, and S. W. Wilson (1984) A new preparation for recording single-channel currents from skeletal muscle. Proc. R. Soc. Lond. [B] 221: 455-464.

Strickholm, A.. (1961) Impedance of a small electrically isolated area of the muscle cell surface. J. Gen. Physiol. 44: 1073-1088.

Stühmer, W., and W. Almers (1982) Photobleaching through glass micropipettes: Sodium channels without lateral mobility in the sarcolemma of frog muscle. Proc. Natl. Acad. Sci. USA 79: 946-950.

Stühmer, W., W. M. Roberts, and W. Almers (1983) The loose patch clamp. In Single-channel Recording, B. Sakmann and E. Neher, eds., pp. 123-132, Plenum, New York.

Tank, D. W., E. S. Wu, and W. W. Webb (1982) Enhanced molecular diffusibility in muscle membrane blebs: Release of lateral constraints. J. Cell Biol. 92: 207-212.

Yechiel, E., and M. Edidin (1987) Micrometer-scale domains in fibroblast plasma membranes. J. Cell Biol. 105: 755-760.

Zollinger, H. U. (1948) Cytologic studies with the phase microscope. I. The formation of "blisters" on cells in suspension (potocytosis) with observations on the nature of the cellular membrane. $\Lambda \mathrm{m}$. J. Pathol. 24: 545-567. 